\title{
THE RADON-NIKODYM THEOREM FOR MULTIMEASURES
}

\author{
by LE VAN TU \\ (Received 14th March 1977)
}

\section{Introduction}

Let $(S, \mathcal{M})$ be a measurable space (that is, a set $S$ in which is defined a $\sigma$-algebra $\mathcal{M}$ of subsets) and $X$ a locally convex space. A map $M$ from $\mathcal{M}$ to the family of all non-empty subsets of $X$ is called a multimeasure iff for every sequence of disjoint sets $A_{n} \in \mathcal{M}(n=1,2, \ldots)$ with $\cup_{n=1}^{\infty} A_{n}=A$, the series $\sum_{n=1}^{\infty} M\left(A_{n}\right)$ converges (in the sense of $(6)$, p. 3) to $M(A)$.

The concept of multimeasure with values in $\mathbf{R}^{n}$ was first introduced by Vind $(15, p$. $174)$ in order to solve some problems in economics. In (14, Théorème 23, p. 292), Valadier has proved the Radon-Nikodym theorem for multimeasures taking values in $\mathbf{R}^{n}$ (or $\mathbf{R}^{\infty}$ ) using the notion of scalar integrability of set-valued functions. (For further results in this aspect, see (3) and (11).)

In Section 2 of this paper, we shall define integrability for a special class of set-valued functions, which we shall call perfectly measurable multifunctions. Then we prove a theorem (Theorem 1) that serves as an example of a multimeasure. In Section 3 we prove the main result, the Radon-Nikodym theorem for multimeasures taking values in a locally convex space; this, however, is not a generalisation of Théorème 23 of (14), nor a consequence of that.

I should like to express my gratitude to Professor A. P. Robertson for suggesting this problem and for many helpful discussions.

\section{Integrable multifunctions}

Henceforth, $(S, \mu)$ is a measurable space, $\mu$ is a finite positive measure on $\mathcal{M}$ and $X$ is a Hausdorff locally convex space with (topological) dual $X^{\prime}$, except where otherwise specified.

Let $F$ be a map that assigns to each $t \in S$, a non-empty set $F(t) \subseteq X$. Then $F$ is called a multifunction (or a set-valued function) from $S$ to $X$. A point-valued function $f$ from $S$ to $X$ is called a selector for $F$ iff $f(t) \in F(t)$ for every $t \in S$. For any subset $B$ of $X$, we put

$$
F^{-1}(B)=\{t \in S: F(t) \cap B \neq \phi\} .
$$

The multifunction $F$ is called measurable iff $F^{-1}(B) \in \mathcal{M}$ for each closed subset $B$ of $X$. We say that $F$ is perfectly measurable iff it is measurable and, for every closed subset $B$ of $X$, the multifunction $F_{B}$ (called the refinement of $F$ by $B$ ), defined on $F^{-1}(B)$ by $F_{B}(t)=F(t) \cap B$, has a measurable selector.

The following Lemma 1 that assures a measurable selector for $F$ is due to Leese 
(7) (for other results on the existence of measurable selectors for a multifunction, we refer to (1), (4), (10) and (12)). Because this work does not yet seem to have been published, a brief proof is included.

Lemma 1. Suppose that $X^{\prime}$ contains a sequence $\left(x_{n}^{\prime}\right), n=1,2, \ldots$, which separates the points of $X$. Then every compact-valued measurable multifunction $F$ from $S$ to $X$ has a measurable selector.

Proof. For each $t \in S$, let $F_{0}(t)=F(t)$ and define $F_{n}(t)(n=1,2, \ldots)$ inductively as follows

$$
F_{n}(t)=\left\{x \in F_{n-1}(t):\left\langle x, x_{n}^{\prime}\right\rangle \text { maximal }\right\} .
$$

Then it can be shown that each $F_{n}$ is a compact-valued measurable multifunction from $S$ to $X$. Moreover, it is clear that $\cap_{n=1}^{\infty} F_{n}(t)$ consists of a single point, $f(t)$ say, and that for every closed set $B$ in $X$,

$$
f^{-1}(B)=\bigcap_{n=1}^{\infty} F_{n}^{-1}(B) .
$$

Therefore $f$ is a measurable selector for $F$.

Lemma 2. Suppose that $X^{\prime}$ contains a sequence which separates the points of $X$. Then every compact-valued measurable multifunction $F$ from $S$ to $X$ is perfectly measurable.

Proof. Let $B$ be a closed subset of $X$. Let $F_{B}$ be the refinement of $F$ by $B$, which is defined on $F^{-1}(B)$ by $F_{B}(t)=F(t) \cap B$. Then for every closed set $C$ in $X$,

$$
F_{B}^{-1}(C)=F^{-1}(B \cap C) \text {, }
$$

which is measurable. Thus $F_{B}$ is a compact-valued measurable multifunction from $F^{-1}(B)$ to $X$. Hence, by Lemma $1, F_{B}$ has a measurable selector. Therefore $F$ is perfectly measurable.

Before going on, let us recall that a (point-valued) function $f$ from $(S, \mathcal{M}, \mu)$ to $X$ is called scalarly integrable iff for every $x^{\prime} \in X^{\prime}$, the function $\left\langle x^{\prime}, f\right\rangle=x^{\prime} \circ f$ is integrable. Then, for any $A \in \mathcal{M}$, we denote by $\int_{A} f d \mu$ the linear form on $X^{\prime}$ defined by

$$
\left\langle x^{\prime}, \int_{A} f d \mu\right\rangle=\int_{A}\left\langle x^{\prime}, f\right\rangle d \mu .
$$

A measurable function $f$ from $(S, \mu, \mu)$ to $X$ is said to be integrable iff $f$ is scalarly integrable and $\int_{A} f d \mu \in X$ for every $A \in \mathcal{M}$ (see for example (9)).

Now let $F$ be a multifunction from $(S, \mathcal{M}, \mu)$ to $X$ and let $\mathscr{S}(F)$ denote the set of all measurable selectors of $F$. The multifunction $F$ is called integrable iff $F$ is perfectly measurable and every $f \in \mathscr{S}(F)$ is integrable. We denote, for any $A \in \mathcal{M}$,

$$
\int_{A} F d \mu=\left\{\int_{A} f d \mu: f \in \mathscr{P}(F)\right\}
$$

which is a subset of $X$. Note that we require $F$ to be perfectly measurable so that 
every refinement of $F$ (by any closed subset $B$ of $X$ ) contributes to the integral (of course provided that $A \cap F^{-1}(B)$ has a non-zero measure). Otherwise, it may happen that $F(t)=G(t) \cup\{x\}$ for each $t \in S$, where $G(t)$ is contained in a fixed closed subset $B$ of $X, G$ has no measurable selector, and $x \in X \backslash B$. In such a case, the integral of $F$ does not reflect the full range of values taken by $F$ at all (For the basic properties of integrable multifunctions, see (13)).

Theorem 1. Let $F$ be an integrable multifunction from $S$ to $X$. Then the set-valued map $M$ from $\mathcal{M}$ to $X$, defined by

$$
M(A)=\int_{A} F d \mu \quad(A \in \mathcal{M})
$$

is a multimeasure.

Proof. Let $\left(A_{n}\right), n=1,2, \ldots$, be a sequence of disjoint sets in $\mathcal{M}$ and let $A=\bigcup_{n=1}^{\infty} A_{n}$. We prove that

$$
M(A)=\sum_{n=1}^{\infty} M\left(A_{n}\right)
$$

For each $n$, let $x_{n} \in M\left(A_{n}\right)$. Then there exist $f_{n} \in \mathscr{S}(F)$ such that $x_{n}=\int_{A_{n}} f_{n} d \mu$, $n=1,2, \ldots$. Let us define a function $f$ by

$$
f=\left\{\begin{array}{l}
f_{n} \text { on } A_{n}, \quad n=1,2, \ldots \\
f_{1} \text { on } S \backslash A .
\end{array}\right.
$$

Certainly $f \in \mathscr{S}(F)$, hence $f$ is integrable and $x_{n}=\int_{A_{n}} f d \mu$. Now, for every $x^{\prime} \in X^{\prime}$ and every positive integer $N$,

$$
\left\langle x^{\prime}, \sum_{n=1}^{N} x_{n}\right\rangle=\sum_{n=1}^{N} \int_{A_{n}}\left\langle x^{\prime}, f\right\rangle d \mu,
$$

which converges, as $N \rightarrow \infty$, to

$$
\int_{\mathrm{A}}\left\langle x^{\prime}, f\right\rangle d \mu=\left\langle x^{\prime}, \int_{A} f d \mu\right\rangle .
$$

This means that the series $\sum_{n=1}^{\infty} x_{n}$ converges weakly to $x=\int_{A} f d \mu$, and a similar property holds for every subseries of $\sum_{n=1}^{\infty} x_{n}$. Hence, by the Orlicz-Pettis Theorem (see for example (6), p. 4), the series $\Sigma_{n=1}^{\infty} x_{n}$ converges (unconditionally) to $x$, which belongs to $M(A)$. Thus we have proved that the series $\Sigma_{n=1}^{\infty} M\left(A_{n}\right)$ is (unconditionally) convergent and is contained in $M(A)$.

To prove the reverse inclusion let $x \in M(A)$; then $x=\int_{A} f d \mu$ for some $f \in \mathscr{S}(F)$. Then, as before, the series $\sum_{n=1}^{\infty} \int_{A_{n}} f d \mu$ converges to $x$. This shows that $x \in$ $\sum_{n=1}^{\infty} M\left(A_{n}\right)$, and completes the proof.

\section{The Radon-Nikodym theorem}

Let $K$ be a convex closed subset of $X$ and let $x^{\prime} \in X^{\prime}$. We write $\varphi\left(x^{\prime}, K\right)=$ $\sup \left\{\left\langle x^{\prime}, x\right\rangle: x \in K\right\}$. Following Meyer (8, p. 32), we denote by $\mathscr{L}^{\infty}(S, \mathcal{M})$ (resp. $\mathscr{L}^{\prime}(S, \mathcal{M}, \mu)$ ) the vector space of all measurable bounded (resp. integr able) real-valued 
functions on $S$ and by $L^{\infty}(S, \mu, \mu)$ (resp. $L^{\prime}(S, \mu, \mu)$ ) the associated quotient space under the relation of equality $\mu$-almost everywhere.

We first prove the following lemma.

Lemma 3. Suppose that $X$ is semireflexive. Let $\rho$ be a real-valued function, defined on $X^{\prime}$, satisfying:

(i) $\rho\left(x^{\prime}+y^{\prime}\right) \leqslant \rho\left(x^{\prime}\right)+\rho\left(y^{\prime}\right)$ and $\rho\left(\lambda x^{\prime}\right)=\lambda \rho\left(x^{\prime}\right)$ for $\lambda \geqslant 0$,

(ii) for every $\epsilon>0, \rho^{-1}((-\infty, \epsilon))$ is a neighbourhood of 0 in $X^{\prime}$.

Then $\rho$ is $\sigma\left(X^{\prime}, X\right)$-lower semicontinuous.

Proof. Let $\alpha \in R$; we prove that the set

$$
A=\left\{x^{\prime} \in X^{\prime}: \rho\left(x^{\prime}\right) \leqslant \alpha\right\}
$$

is $\sigma\left(X^{\prime}, X\right)$-closed. Since $X$ is semireflexive and $A$ is convex, it is sufficient to prove that $A$ is strongly closed. Let $y^{\prime} \in \bar{A}$ and let $\epsilon>0$. By (ii), there exists a balanced neighbourhood $U$ of 0 in $X^{\prime}$ such that $z^{\prime} \in U$ implies $\rho\left(z^{\prime}\right)<\epsilon$. Then there is $x^{\prime} \in y^{\prime}+U$ such that $\rho\left(x^{\prime}\right) \leqslant \alpha$. It follows that

$$
\rho\left(y^{\prime}\right) \leqslant \rho\left(y^{\prime}-x^{\prime}\right)+\rho\left(x^{\prime}\right)<\epsilon+\alpha .
$$

Therefore $y^{\prime} \in A$, which completes the proof.

Theorem 2. Let $(S, \mu, \mu)$ be a probability space (i.e. $\mu(S)=1)$ and $X$ a locally convex space that is semireflexive. Assume that $X^{\prime}$ contains a sequence which separates the points of $X$. Also let $M$ be a convex compact-valued multimeasure from $\mathcal{M}$ to $X$. Suppose that there exist a convex compact metrizable subset $K$ of $X$ and a positive measure $\nu \ll \mu$ such that for every $A \in \mathcal{M}$,

$$
M(A) \subseteq \nu(A) K .
$$

Then there is a convex compact-valued integrable multifunction $F$ from $S$ to $X$ such that

$$
M(A)=\int_{A} F d \mu,
$$

for every $A \in \mathcal{M}$.

Proof. We may suppose that $K$ is balanced without loss of generality. For every $x^{\prime} \in X^{\prime}$, we define for each $A \in \mathcal{M}$,

$$
\mu_{x^{\prime}}(A)=\varphi\left(x^{\prime}, M(A)\right)
$$

Then each $\mu_{x^{\prime}}$ is a real-valued bounded measure and these measures satisfy the following properties:

(i) $\mu_{x^{\prime}+y^{\prime}} \leqslant \mu_{x^{\prime}}+\mu_{y^{\prime}}$

(ii) $\mu_{\lambda x^{\prime}}=\lambda \mu_{x^{\prime}}$ for $\lambda \geqslant 0$.

Moreover, for each $x^{\prime} \in X^{\prime}$, we have $\mu_{x^{\prime}} \ll \mu$; hence there is $\psi_{x^{\prime}} \in L^{\prime}(S, \mu, \mu)$ such that for every $A \in \mathcal{M}$,

$$
\mu_{x^{\prime}}(A)=\int_{A} \psi_{x^{\prime}} d \mu .
$$


Certainly the functions $\psi_{x^{\prime}}$ satisfy the conditions similar to (i) and (ii).

Now we want to find, for each $x^{\prime} \in X^{\prime}$, a function $\Psi_{x^{\prime}}$ in the class $\psi_{x^{\prime}}$ such that for every $t \in S$, the map $x^{\prime} \rightarrow \Psi_{x^{\prime}}(t)$ satisfies the conditions of Lemma 3 . Let $\theta$ be the density function of $\nu$ with respect to $\mu$. For every $x^{\prime} \in X^{\prime}$ and every $A \in \mathcal{M}$, since $M(A) \subseteq \nu(A) K$, we have

$$
\varphi\left(x^{\prime}, M(A)\right) \leqslant \varphi\left(x^{\prime}, \nu(A) K\right)=\nu(A) \varphi\left(x^{\prime}, K\right) .
$$

Hence, putting $k_{x^{\prime}}=\left|\varphi\left(x^{\prime}, K\right)\right|$, we have $\left|\mu_{x^{\prime}}\right|(A) \leqslant \nu(A) k_{x^{\prime}}$, for every $A \in \mathcal{M}$. Therefore, for every $x^{\prime} \in X^{\prime}$,

$$
\left|\psi_{x^{\prime}}\right| \leqslant k_{x^{\prime}} \theta
$$

Let us choose a non-negative member $\Theta$ in the class $\theta$ and put, for $n=0,1,2, \ldots$,

$$
S_{n}=\{t \in S: n \leqslant \Theta(t)<n+1\} \text {. }
$$

Thus each $S_{n} \in \mathcal{M}$ and the $S_{n}$ form a partition for $S$. For each $n=0,1,2, \ldots$ let $\psi_{x^{\prime}, n}$ be the restriction of $\psi_{x^{\prime}}$ on $S_{n}$ and define $\mathcal{M}_{n}, \mu_{n}$ analogously. Then $\psi_{x^{\prime}, n} \in$ $L^{\infty}\left(S_{n}, \mathcal{M}_{n}, \mu_{n}\right)$. Therefore, by the Lifting Theorem (8, Théorème 12, p. 195), each $\psi_{x^{\prime}, n}$ can be lifted to a function $\Psi_{x^{\prime}, n} \in \mathscr{L}^{\infty}\left(S_{n}, \mathcal{M}_{n}\right)$ (note that the lifting map is linear, positive and isometric). We obtain the function $\Psi_{x^{\prime}}$ by gluing the functions $\Psi_{x^{\prime}, n}$ together. It is clear that $\Psi_{x^{\prime}} \in \mathscr{L}^{\prime}(S, \mathcal{M}, \mu)$ and

(iii) $\Psi_{x^{\prime}+y^{\prime}} \leqslant \Psi_{x^{\prime}}+\Psi_{y^{\prime}}$,

(iv) $\Psi_{\lambda x^{\prime}}=\lambda \Psi_{x^{\prime}}$ for $\lambda \geqslant 0$.

Now, let $t$ be chosen and fixed in $S$; then $t \in S_{n}$ for some $n=0,1,2, \ldots$ For every $x^{\prime} \in X^{\prime}$, since $\left\|\psi_{x^{\prime}, n}\right\|_{\infty} \leqslant k_{x^{\prime}}(n+1)$, we have $\left\|\Psi_{x^{\prime}, n}\right\| \leqslant k_{x^{\prime}}(n+1)$ and hence

$$
\left|\Psi_{x^{\prime}}(t)\right| \leqslant k_{x^{\prime}}(n+1) \text {. }
$$

According to Hörmander (5, Théorème 7), the function $x^{\prime} \rightarrow k_{x^{\prime}}$ is (strongly) continuous. Hence the function $x^{\prime} \rightarrow \Psi_{x^{\prime}}(t)$ is continuous at 0 . This fact, combined with (iii) and (iv), implies that the function $x^{\prime} \rightarrow \Psi_{x^{\prime}}(t)$ is $\sigma\left(X^{\prime}, X\right)$-lower semicontinuous (Lemma 3). Therefore (by Théorème 5 of $(5)$ ), there is a convex closed subset $F(t)$ of $X$ such that

$$
\Psi_{x^{\prime}}(t)=\varphi\left(x^{\prime}, F(t)\right),
$$

for every $x^{\prime} \in X^{\prime}$. Moreover if $x^{\prime} \in K^{\circ}$, the polar set of $K$, then $k_{x^{\prime}} \leqslant 1$. It follows that

$$
F(t) \subseteq(n+1) K^{\infty}=(n+1) K .
$$

Hence $F(t)$ is compact for each $t \in S$.

Next, we prove that the multifunction $F$ is integrable. Note first that for every $x^{\prime} \in X^{\prime}$, the function $t \rightarrow \varphi\left(x^{\prime}, F(t)\right)$ is measurable and that $F(t)$ is contained in the convex compact metrizable set $(n+1) K$ whenever $t \in S_{n}$. Thus (by Proposition 8 of (14)), the restriction of $F$ on each $S_{n}(n=0,1,2, \ldots)$ is measurable. Therefore $F$ is measurable. Then, by Lemma $2, F$ is perfectly measurable. Now let $f \in \mathscr{P}(F)$; then for every $x^{\prime} \in X^{\prime}$,

$$
-\Psi_{-x^{\prime}} \leqslant\left\langle x^{\prime}, f\right\rangle \leqslant \Psi_{x^{\prime}}
$$

This shows that $f$ is scalarly integrable. Furthermore, for each $n=0,1,2, \ldots, f\left(S_{n}\right) \subseteq$ 
$(n+1) K$ which is convex compact and balanced. Therefore (by Théorème 1 of $(2)), f$ is integrable (that is, $\int_{A} f d \mu \in X$ for every $A \in \mathcal{M}$ ). This means that $F$ is integrable.

Finally, since $X$ is semireflexive and because every scalarly measurable selector of $F$ is measurable, we obtain from (2, Théorème 2$)$

$$
\varphi\left(x^{\prime}, \int_{A} F d \mu\right)=\int_{A} \varphi\left(x^{\prime}, F(\cdot)\right) d \mu,
$$

for every $A \in \mathcal{M}$ and every $x^{\prime} \in X^{\prime}$. Yet the right-hand side is the same as

$$
\int_{A} \Psi_{x^{\prime}} d \mu=\int_{A} \psi_{x^{\prime}} d \mu=\varphi\left(x^{\prime}, M(A)\right)
$$

Therefore, by Théorème 1 of (5),

$$
M(A)=\int_{A} F d \mu,
$$

for every $A \in \mathcal{M}$. This completes the proof.

\section{REFERENCES}

(1) C. Castaing, Sur les multiapplications mesurables, Rev. Française Inform. Recherche Opérationnelle 1 (1967), 91-126.

(2) C. CAStaing and M. VAladier, Équations différentielles multivoques dans les espaces vectoriels localement convexes, Rev. Française Inform. Recherche Opérationnelle 16 (1969), 3-16.

(3) A. Costé, Applications de la théorie des probabilités cylindriques et des opérateurs radonifiants à l'étude des fonctions aléatoires sous-linéaires et des multimesures, C.R. Acad. Sci. Paris, 282, Série A (1976), 103-106.

(4) C. J. Himmelberg and F. S. VAN VLECK, Some selection theorems for measurable functions, Canadian J. Math. 21 (1969), 394-399.

(5) L. Hörmander, Sur la fonction d'appui des ensembles convexes dans un espace localement convexe, Arkiv för Mat. 3 (1954), 181-186.

(6) I. KLUVÁNEK and G. KNOWLES, Vector measures and control systems (North-Holland, Amsterdam, 1975).

(7) S. J. LeESE, Set-valued functions and selectors, (Ph.D. thesis, University of Keele, 1974).

(8) P. A. Meyer, Probabilités et potentiel (Hermann, Paris, 1966).

(9) B. J. PeTtis, On integration in vector spaces, Trans. Amer. Math. Soc. 44 (1938), 277-304. $1-7$.

(10) A. P. Robertson, On measurable selections, Proc. Roy. Soc. Edinburgh, (A) 72 (1974),

(11) A. A. Tolstonogov, On the theorems of Radon-Nikodym and A. A. Ljapunov for a multivalued measure, Soviet Math. Dokl., 16 (1975), 1588-1592 (Dokl. Akad. Nauk SSSR, 225 (1975), 1023-1026). 
(12) L. V. TU, Omega-Polish spaces and measurable selections, J. Australian Math. Soc. (Series A) 23 (1977), 257-265.

(13) L. V. TU, On multifunctions and multimeasures, (Ph.D. thesis, Murdoch University, February 1977).

(14) M. V ALADIER, Multiapplications mesurables à valeurs convexes compactes, J. Math. pures et appl., 50 (1971), 265-297.

(15) K. VIND, Edgeworth-allocations in an exchange economy with many traders, Internat. Econ. Review 5 (1964), 165-177.

UNIVERSITY OF WESTERN AUSTRALIA,

NEDLANDS, W.A. 6009.

AUSTRALIA. 